\title{
Ethische Fragen ohne einfache Antworten
}

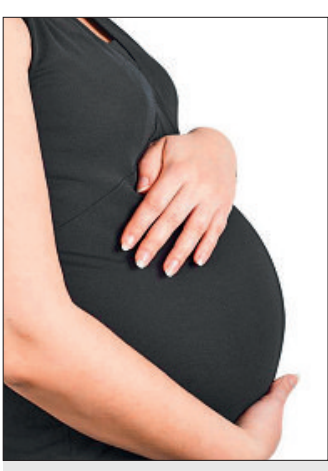

Kaiserschnitt auf Wunsch? Kaiserschnitt auf Anordung? Beide Positionen beinhalten erhebliche ethische Herausforderungen.

1 Martin J. Biomédecine et procréation. Revue médicale suisse. 2005; 1:453-8.

2 Les césariennes de la discorde. 24 Heures (Lausanne), 21 décembre 2010. p. 33.

3 Martin J. Können Patienten auf ärztlichen Leistungen bestehen? Schweiz Ärztezeitung. 2010, 91(38):1504.

4 Minkoff H, Drapkin Lyerly A. Samantha Burton and the rights of pregnant women. Hastings Center Report. Nov./Dec. 2010; 40. No. 6. p. 13-5.

5 Kolder VE, Gallagher J, Parsons MT. Court-ordered obstetrical interventions. New England Journal of Medicine. 1987; 316:1192-6.

6 Appel JM. Medical kidnapping: rogue obstetricians vs. pregnant women. Huffington Post. January 24; 2010.

7 Da mir diese Frage gelegentlich von Studierenden oder Kollegen/-innen gestellt wird, möchte ich bei dieser Gelegenheit betonen, dass es andererseits in unseren Rechtsordnungen keine Situation gibt, in der eine Frau zu einem Schwangerschaftsabbruch gezwungen werden kann.
Jede dritte Schwangerschaft in der Schweiz wird heute mit einem Kaiserschnitt beendet. $\mathrm{Zu}$ meiner Studienzeit lag die Zahl bei unter 10\%. Die Zeiten ändern sich, die Medizin entwickelt sich, und alle Fortschritte sollen den Patientinnen und Patienten zugute kommen, um bestmögliche Lebensqualität und geringstmögliche Beschwerden zu gewähren. Dennoch stellt sich die Frage, wie weit sich die neuen technisierten Methoden der Kinderzeugung ausdehnen werden [1].

Hebammen-Organisationen reagieren alarmiert auf diese Abkehr von der natürlichen Geburt. Ich erinnere mich an Plakate englischer Kliniken, auf denen mit dem Slogan «Saving the love channel» für den Kaiserschnitt geworben wurde. Kürzlich musste ich zu meiner grossen Überraschung erfahren, dass in einer deutschen Universitätsklinik dem Wunsch der Patientin nach Kaiserschnitt stattgegeben wird, wenn sie darauf besteht, auch wenn die medizinische Notwendigkeit fehlt. Dazu ein übersetzter Auszug aus einem Interview in 24 Heures: «Bevor ich schwanger wurde, habe ich mich vergewissert, dass eine Geburt per Kaiserschnitt möglich ist. Ausgeschlossen, dass ich ein Kind vaginal auf die Welt bringen würde.» Einige vergleichen die vaginale Entbindung mit einer Art grausamem Ritual [2]! Nun ... ich muss gestehen, dass ich merke, einer älteren Generation anzugehören. Zwar habe ich mich stets für die Anerkennung und gesetzliche Verankerung der Patientenrechte stark gemacht, aber es scheint sich immer mehr die Frage aufzudrängen, ob die Patienten auch das Recht haben, Leistungen von ihrem Arzt zu fordern [3].

Dieselbe Frage, nämlich Kaiserschnitt Ja oder Nein, kann umgekehrt gestellt werden. Sie wird in der jüngsten Ausgabe der Zeitung für Bioethik, dem Hastings Center Report, diskutiert [4]. In diesem Fall geht es darum, ob und wann es gerechtfertigt ist, dass ein Gericht die Entbindung per Kaiserschnitt gegen den Willen der Frau anordnet. Ich habe mich aus meiner Sicht als Kantonsarzt mit dieser Frage befasst, denn in einigen US-Staaten besteht die Möglichkeit, z.B. bei drogenabhängigen Frauen, die kurz vor der Entbindung stehen, einen Kaiserschnitt gerichtlich anzuordnen, wenn eine schwere gesundheitliche Gefährdung des Kindes zu befürchten ist [5]. Auf unserer Seite des Atlantiks gestattet allerdings kein Land einen derartigen Eingriff in die körperliche Integrität, wenn die betroffene Person dies ablehnt - auch zum Wohle des ungeborenen Kindes nicht.

Dazu eine weitere Anmerkung: Kolder et al. haben festgestellt, dass am häufigsten Frauen zu einem Kaiserschnitt gezwungen wurden, die aus armen Verhält- nissen stammten, Minderheiten angehörten und häufig kein Englisch sprachen. Dies ist ein weiteres Beispiel für eine Konstante in Public Health: Die den Menschen zugänglichen Gesundheitsleistungen hängen von ihrem Status ab und die sozio-ökonomischen Disparitäten finden ihren Ausdruck in Ungleichbehandlung (dies gilt im übrigen ebenfalls für die Wunsch-Schnittentbindung, die wahrscheinlich häufiger Frauen aus der mittleren oder gehobenen Schicht gewährt wird).

Minkoff und Dryerly beschreiben, dass in Sachen des «Zwangskaiserschnitts» in den USA derzeit eine Gegenbewegung zu beobachten ist, und führen mehrere Berufungsfälle an, in denen höhere Gerichte der Rechtfertigung eines angeordneten Kaiserschnitts widersprachen. Ihre Sorge ist allerdings, dass, obgleich die Geburtshelfer das Recht der Frauen auf Ablehnung medizinischer Massnahmen respektieren, diese Rechte in den letzten zwei Jahrzehnten ausgehöhlt worden sind. Dafür gebe es unter anderem zwei Gründe: den unaufhörlichen Kampf um die Abtreibung und die Einstellung, dass Frau und Fötus in einem grundsätzlichen Konfliktverhältnis zueinander stünden. Nach Ansicht der Autoren würden «die Rechte schwangerer Frauen heutzutage häufig mehr beschnitten als die Rechte von Eltern an ihren bereits geborenen Kindern». Dies erinnert an einen Gedanken, der einst in den Debatten um die Fristenregelung zu hören war. Man fragte sich, ob diejenigen, die sich grundsätzlich gegen einen Schwangerschaftsabbruch aussprachen, auch bereit wären, ebenso viel Energie und Mittel aufzuwenden, um sich angemessen um die in desolate Verhältnisse hineingeborenen Kinder zu kümmern.

Zum Abschluss ein Rat eines anderen amerikanischen Autors an die Frauen: Sie sollten ihrem Gynäkologen einmal die Frage stellen, ob es Umstände geben könnte, unter denen er ihnen verweigern würde, selbst über eine mögliche Behandlung zu entscheiden [6]. Interessante Frage; ich vertrete die Ansicht - wie sicherlich alle, die sich mit ethischen Fragen befassen - dass das Recht der Frau auf Ablehnung eines Kaiserschnitts unteilbar und massgebend ist, unabhängig davon, wer diesen Eingriff anordnen sollte [7]; dagegen bin ich im Hinblick auf ihr Recht, einen Kaiserschnitt fordern zu können, skeptisch und fühle mich bei dem Gedanken unwohl. Sollte ich mich einmal selbst befragen, ob ich in meiner Haltung von patriarchalischen oder chauvinistischen Tendenzen geleitet bin?

\section{Jean Martin, Mitglied der Nationalen Ethikkommission} und Mitglied der Redaktion der SÄZ 